\title{
Direct detection of aptamer-thrombin binding via surface-enhanced Raman spectroscopy
}

\section{Cynthia V. Pagba}

University of California, Davis

National Science Foundation

Center for Biophotonics Science and Technology

2700 Stockton Boulevard, Suite 1400

Sacramento, California 95817

\section{Stephen M. Lane}

University of California, Davis

National Science Foundation

Center for Biophotonics Science and Technology and

Department of Neurological Surgery

2700 Stockton Boulevard, Suite 1400

Sacramento, California 95817

\section{Hansang Cho}

University of California, Davis

National Science Foundation

Center for Biophotonics Science and Technology

2700 Stockton Boulevard, Suite 1400

Sacramento, California 95817 and

Lawrence Livermore National Laboratory

Physical and Life Sciences

7000 East Avenue

Livermore, California 94550

and

University of California, Berkeley

Department of Bioengineering

UCSF/UCB Joint Graduate Group in Bioengineering

Biomolecular Nanotechnology Center

Berkeley Sensor and Actuator Center

Berkeley, California 94720

\section{Sebastian Wachsmann-Hogiu}

University of California, Davis

National Science Foundation

Center for Biophotonics Science and Technology and

Department of Neurological Surgery

2700 Stockton Boulevard, Suite 1400

Sacramento, California 95817

\begin{abstract}
In this study, we exploit the sensitivity offered by surfaceenhanced Raman scattering (SERS) for the direct detection of thrombin using the thrombin-binding aptamer (TBA) as molecular receptor. The technique utilizes immobilized silver nanoparticles that are functionalized with thiolated thrombin-specific binding aptamer, a 15-mer (5'-GGTTGGTGTGGTTGG-3') quadruplex forming oligonucleotide. In addition to the Raman vibrational bands corresponding to the aptamer and blocking agent, new peaks (mainly at 1140, 1540, and $1635 \mathrm{~cm}^{-1}$ ) that are characteristic of the protein are observed upon binding of thrombin. These spectral changes are not observed when the aptamer-nanoparticle assembly is exposed to a nonbinding protein such as bovine serum albumin (BSA). This methodology could be further used for the development of label-free biosensors for direct detection of proteins and other molecules of interest for which aptamers are available. (๑) 2010 Society of Photo-Optical Instrumentation Engineers. [DOI: $10.1117 / 1.3465594]$
\end{abstract}

Keywords: aptamer; quadruplex; label-free assay; direct detection; thrombin; surface-enhanced Raman Scattering.

Paper 10114R received Mar. 5, 2010; revised manuscript received May 10, 2010; accepted for publication May 18, 2010; published online Aug. 6, 2010.

\section{Introduction}

Detection and tracking of biomolecules is invaluable not only in the field of medical diagnostics but also in biological and biotechnology research, drug development, environmental monitoring, forensic investigations, and biodefense. To this end, researchers of different expertise have sought to develop methodologies/techniques that are sensitive, specific, robust, high throughput (amenable to multiplexing), simple, and cost

Address all correspondence to: Cynthia V. Pagba, University of California Davis, 2700 Stockton Boulevard, Suite 1400, Sacramento, California 95817; Tel: 916-734-0784; E-mail: cvpagba@ucdavis.edu; or Sebastian WachsmannHogiu, University of California, Davis, 2700 Stockton Boulevard, Suite 1400 Sacramento, California 95817; Tel: 916-734-1774; E-mail: swachsmann@ucdavis.edu. effective. Presently, most methods used to detect biomolecules that require molecular recognition events (e.g., formation of antibody-antigen or aptamer-analyte complexes) are variations of the "sandwich type" enzyme-linked immunosorbent assays (ELISA) and typically rely on the use of receptors tagged with probe molecules, which may not always be desirable. ${ }^{1-3}$ Labeling of biomolecules is a time-consuming process that can lead to the loss of biological activity. Other assays make use of nanoparticles and their tendency to form aggregates, which results in color change due to a shift in the plasmon resonance in response to binding events. ${ }^{4,5}$ While this is a convenient method, it is limited by its susceptibility

1083-3668/2010/15(4)/047006/8/\$25.00 (C) 2010 SPIE 
to changes in the environment, e.g., change in ionic strength or $\mathrm{pH}$, which can also induce aggregation and color change. Another nanoparticle-based technique takes advantage of the ability of metal nanoparticles to quench emission from nearby fluorescent compounds. In this case, the presence of the analyte is detected by the change in the intensity of emission from the fluorescent molecule attached to the receptor. ${ }^{6,7} \mathrm{Di}$ rect detection techniques such as mass spectrometry and surface plasmon resonance (SPR) spectroscopy are also widely used, but they are expensive and potentially limited by extensive sample preparation. For example, mass spectrometry requires isolation and purification of the analyte (usually by chromatography). ${ }^{8}$ SPR, which detects binding of the molecule by measuring the change in the refractive index at the surface of the metal functionalized with a conjugated receptor, is subject to nonspecific interactions.

Another technique that is currently under development is based on surface-enhanced Raman scattering (SERS). SERS occurs when a molecule is in close proximity to the metal surface. When combined with the resonance Raman effect, signal enhancements of up $10^{14}$ to $10^{15}$ have been observed, allowing even single molecule detection ${ }^{9}$ under very favorable conditions. This huge signal enhancement is attributed to enhanced electromagnetic fields within the immediate vicinity of the metal upon excitation of plasmon resonances by photon interaction, ${ }^{10}$ and to charge transfer processes between the metal and the adsorbed molecule. ${ }^{11}$

SERS-based assays, ${ }^{3,12-14}$ which require molecular recognition events, have been made possible by labeling either the capture agent or the target molecule itself with a Ramanactive molecule. Recently, Neuman et al. ${ }^{15}$ reported SERSbased direct detection of the platelet derived growth factor (PDGF) by monitoring the randomness of the SERS spectra of the PDGF aptamer, following its exposure to the target molecule PDGF. The change in the reproducibility of the pristine anti-PDGF aptamer was thought to be due to change in the conformation of the aptamer as a result of protein binding.

Aptamers are functional single-stranded oligonucleotides (DNA or RNA) generated by the process called systematic evolution of ligands by exponential enrichment (SELEX). ${ }^{16}$ They bind to their target molecules selectively and with high affinity by forming secondary structures and shapes (e.g., quadruplex, hairpin loop, and T-junction). ${ }^{17,18}$ Several aptamers have been developed for the detection of certain biological and chemical threat agents [e.g., ricin, ${ }^{19}$ anthrax spores, ${ }^{20}$ and trinitrotoluene $\left.(\mathrm{TNT})^{21}\right]$; cancer biomarkers; ${ }^{22-25}$ HIVassociated proteins; ${ }^{26-29}$ food-borne pathogens; ${ }^{30}$ and other biologically important biomolecules such as insulin, ${ }^{31,32}$ immunoglobulin E (IgE), ${ }^{33}$ and thrombin. ${ }^{34}$ They exhibit protein binding affinities that are comparable to those of corresponding antibodies. ${ }^{18,35-38}$ As in the case of antibodies, labeling of the aptamer can sometimes lead to loss of activity resulting from loss of ability to form the secondary structure that is required for binding.

In the present study, we exploit the sensitivity offered by SERS in the development of an aptamer-based directdetection method for target molecules requiring molecular recognition using the thrombin and thrombin binding aptamer $(\mathrm{TBA})^{34}$ pair as a model system. TBA is a 15-mer (5'-GGTTGGTGTGGTTGG-3') oligonucleotide that binds

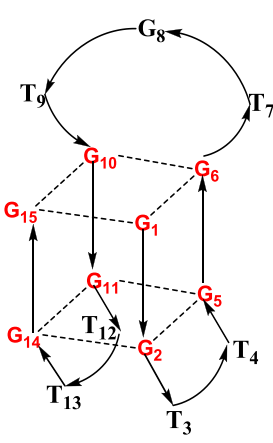

(a)

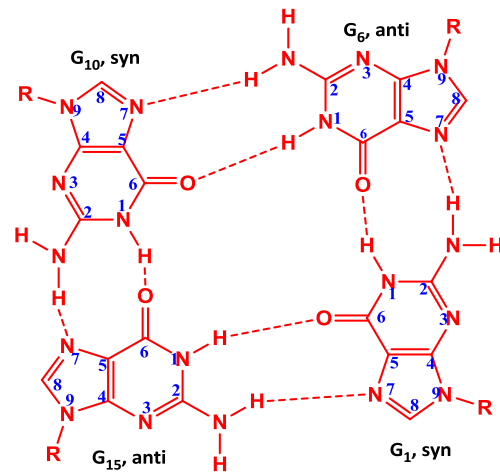

(b)
Fig. 1 (a) Antiparallel quadruplex or chair conformation of the TBA. (b) Guanine quartet of the TBA formed via the Hoogsten-type guanine-guanine interaction. In an antiparallel quadruplex, the guanosine residues have an alternating syn and anti conformation.

to the fibrinogen-recognition exosite of thrombin ${ }^{39}$ after forming a chair-type G-quadruplex structure ${ }^{40-44}$ (Fig. 1). The compact size of the folded aptamer $(\sim 1.1 \mathrm{~nm})^{45}$ makes it an ideal receptor for this assay, as it brings the target molecule in close proximity to the metal surface, i.e., the analyte is well within the required distance $(<10 \mathrm{~nm})$ for local SERS field enhancement.

The technique (Fig. 2) utilizes immobilized nanoparticles that are functionalized with an aptamer. Binding of thrombin is detected by the presence of additional Raman bands following exposure of a TBA-functionalized SERS substrate to the test sample. The method described here may reduce the number of steps required in a sandwiched-based assay, and has the potential to discriminate between specific and nonspecific binding events.

\section{Experimental Section}

The general protocol for the assay is presented in Fig. 2. First, the silver nanoparticles are immobilized on a glass surface previously treated with 3-aminopropyl trimethoxysilane. The immobilized nanoparticles are functionalized with a thiolated thrombin binding aptamer and the surface is blocked with 6-mercaptohexanol to prevent nonspecific binding. A solution containing thrombin is then introduced. SERS spectra are obtained for each step to monitor the relevant binding events. Binding of thrombin is detected by the presence of additional Raman bands following exposure of TBA-functionalized SERS substrate to the test sample.

\subsection{Materials}

Monobasic and dibasic potassium phosphate (KPi) and potassium chloride were obtained from Fisher Scientific. Phosphate buffered saline (PBS) was purchased from USB Corporation, Silver nitrate $\left(\mathrm{AgNO}_{3}\right)$, sodium citrate, (3-aminopropyl) trimethoxysilane (APTMS), 6-mercaptohexanol (MH), bovine serum albumin (BSA), and thrombin were purchased from Sigma Chemical Company (Saint Louis, Missouri). Thrombin was dialyzed (Spectrapor Biotech membrane $3000 \mathrm{MWCO}$ ) against PBS for $3 \mathrm{~h}$ prior to use. The thiolated thrombin binding aptamer $\left(5^{\prime}-\mathrm{HS}-\left(\mathrm{CH}_{2}\right)_{5}-\right.$ $\mathrm{CH}_{2}$-GTTGGTGTGGTTGG-3') was ordered from Sigma 


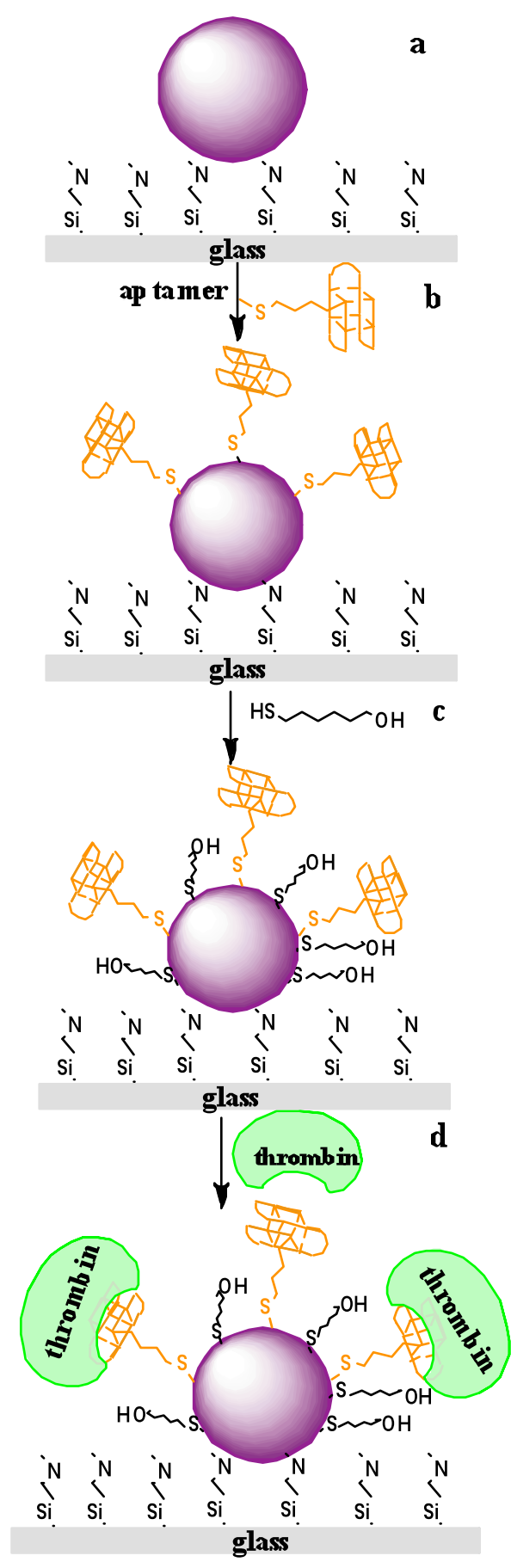

Fig. 2 Scheme for direct detection of thrombin. (a) Nanoparticles are immobilized on a glass surface previously treated with (3aminopropyl) trimethoxysilane. (b) Nanoparticles are functionalized with the thiolated thrombin binding aptamer. (c) The exposed metal surface is blocked by 6-mercaptohexanol. (d) The target molecule (thrombin in this case) is introduced. SERS spectrum is obtained at each step.

Chemical Company. It was supplied as a disulfide form and was used without hydrolysis. Glass cover slips (25CIR-1) were purchased from Fisher Scientific Incorporated (Pittsburgh, Pennsylvania). All glasswares used were cleaned with aqua regia.

\subsection{Silver Nanoparticle Preparation}

Silver nanoparticles (NPs) were prepared following the procedure of Lee and Meisel. ${ }^{46}$ Briefly, $20 \mathrm{mg}$ of $\mathrm{AgNO}_{3}$ was dissolved in $100 \mathrm{~mL}$ of Milli-Q water and the solution was brought to boiling, after which $20 \mathrm{~mL}$ of $0.1 \%$ sodium citrate was added drop-wise. The mixture was then refluxed for one hour. The resulting solution was greenish yellow in color. The plasmon resonance was centered at about $412 \mathrm{~nm}$ as measured by a Cary UV-Vis spectrophotometer (Varian, Palo Alto, California). Functionalization of the nanoparticles resulted in the formation of a new broad absorption band centered at about $660 \mathrm{~nm}$, which allows for SERS measurements at our laser excitation wavelength $(647 \mathrm{~nm})$. The nanoparticles have an average diameter of about $40 \mathrm{~nm}$ as measured by a Nanosight (Amesbury, United Kingdom) LM 20 system and atomic force microscopy.

\subsection{Glass Substrate Pretreatment}

Glass cover slips were cleaned by sonicating them subsequently in acetone, $1 \mathrm{M} \mathrm{NaOH}$, and Milli-Q water for one hour each. They were further rinsed with Milli-Q water three times and dried under nitrogen flow. The cover slips were then treated with freshly prepared $5 \mathrm{mM}$ APTMS (in toluene) for $10 \mathrm{~min}$ and blown dry with nitrogen.

\subsection{Substrate Preparation}

The stock solution of silver nanoparticles was dialyzed against Milli-Q water for $3 \mathrm{~h}$ and was diluted with Milli-Q water to get a $0.2 \mathrm{nM}$ solution. A $10 \mu \mathrm{L}$ aliquot of this solution was deposited on an APTMS- treated glass cover slip and allowed to air dry at room temperature. The cover slip was then washed with Milli-Q water and blown dry with nitrogen flow. A $10 \mu \mathrm{L}$ aliquot of $10 \mu \mathrm{M}$ thrombin binding aptamer in PBS (with $100 \mathrm{mM} \mathrm{KCl}$ added to induce the formation of the quadruplex structure) was added and incubated for $12 \mathrm{~h}$. It was rinsed with Milli-Q water and blown dry with nitrogen. A $0.1 \%$ aqueous solution of 6-mercaptohexanol was then added to block the surface not occupied by the aptamer. The substrate was rinsed with PBS, and $10 \mu \mathrm{L}$ of $10 \mu \mathrm{M}$ thrombin solution was added and incubated at $4{ }^{\circ} \mathrm{C}$ for at least one hour. For the negative control, bovine serum albumin, a nonbinding protein, is used instead of the target molecule.

\subsection{Surface-Enhanced Raman Scattering Measurements}

SERS spectra were acquired using a custom-built Raman system based on a Till Photonics microscope equipped with a $60 \times, 1.45-N A$ oil objective, via a SpectraPro 2300i Acton spectrometer with a Princeton Instruments Pixis100 backilluminated charge-coupled device (CCD) camera, and 647-nm excitation wavelength from an Ar-Kr Innova 70C Coherent laser. An integration time of $60 \mathrm{~s}$ and laser power of $100 \mu \mathrm{W}$ were used for all SERS measurements. The spectra were obtained by taking an average of five measurements from five different clusters/aggregates of nanoparticles. The baseline was corrected using a third-order polynomial fit. 


\section{Results and Discussion}

The use of aptamers as molecular receptors has been attracting a lot of attention for a number of reasons. First, they are less susceptible to denaturation and degradation than the corresponding antibodies. Second, they can be synthesized with high purity and reproducibility and are also easily engineered. Third, they are much smaller than the corresponding antibodies, which is significant in applications where a smaller size of the capture ligand is desired. This is represented in the present case, where the target molecule needs to be in close proximity to the metallic nanoparticle for significant SERS enhancement.

The methodology reported here offers several potential benefits: 1. it does not require biotin-streptavidin binding, as the aptamer can be attached covalently to the SERS substrate via thiolchemistry; 2. the SERS substrate functionalized with aptamer is robust, owing to the stability of the aptamer; and 3. it is also compact and could be further miniaturized to make it suitable for field applications using portable Raman spectrometers.

We have previously reported a Raman and SERS characterization study of both the thiolated and unthiolated analogs of the thrombin binding aptamer (TBA) ${ }^{47}$ We have shown that the formation of the quadruplex structure by TBA, indicated by the diagnostic $\sim 1480-\mathrm{cm}^{-1}$ band $(\mathrm{C} 8=\mathrm{N} 7-\mathrm{H}$ deformation, Fig. 1) ${ }^{48-50}$ under favorable conditions, i.e., presence of $\mathrm{K}+$ and incubation at $\sim 4{ }^{\circ} \mathrm{C}$, can be conveniently monitored by Raman spectroscopy. We also showed that the Raman and SERS spectra of the aptamer are dramatically different. This observation is not uncommon in SERS studies, and can be attributed to different enhancement factors of vibrational modes due to the fact that chemical bonds that are closer to the metallic nanoparticle surface receive stronger enhancement.

For this study, we have used a thiolated analog of the aptamer to facilitate binding of the aptamer to the nanoparticle. To show that thiolation does not prevent formation of the quadruplex structure, we previously measured the Raman spectra of both the thiolated and unthiolated analogs under the same conditions. ${ }^{47}$ Raman spectra of the two analogs both exhibit the characteristic $1480-\mathrm{cm}^{-1}$ band of the quadruplex structure, indicating that thiolation does not inhibit the formation of the quadruplex structure by the aptamer, which is responsible for its binding to thrombin. To ensure that the aptamer is in its quadruplex form before immobilization, it was prepared in PBS containing $\mathrm{K}^{+}$ions.

Figures 3(a) and 3(b) present the individual SERS spectra of the thiolated aptamer (TBA) and the blocking agent mercaptohexanol $(\mathrm{MH})$, respectively. It can be observed that the characteristic $1480-\mathrm{cm}^{-1}$ band of the quadruplex aptamer structure is missing in the SERS spectrum. This may suggest that the aptamer is in its unfolded form, but the absence of the band around $1496 \mathrm{~cm}^{-1}$, which corresponds to the $\mathrm{C} 8=\mathrm{N} 7$ vibration of guanine when it is not hydrogen bonded, ${ }^{50,51}$ indicates otherwise. It is very likely that the guanine tetrads do not interact with the metal, since they are not exposed to it, i.e., their location in the quadruplex configuration does not allow for it. Another very noticeable feature of the SERS spectrum of TBA is the presence of a strong band around $1398 \mathrm{~cm}^{-1}$. This can be attributed mostly to the $\mathrm{CH}_{2}$

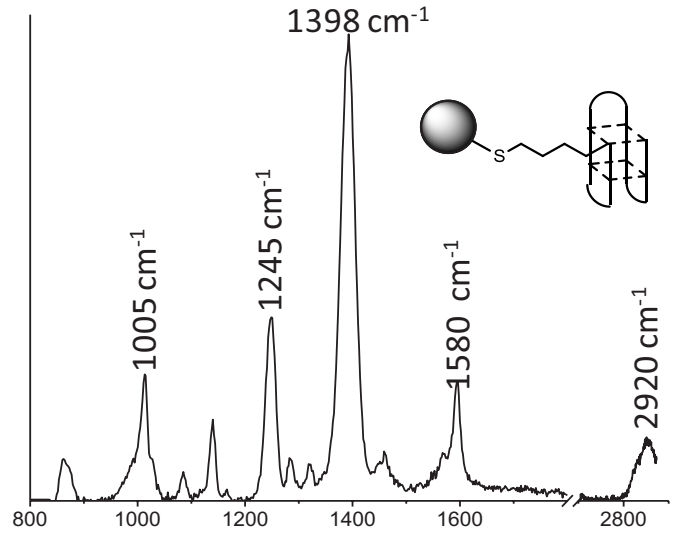

(a)

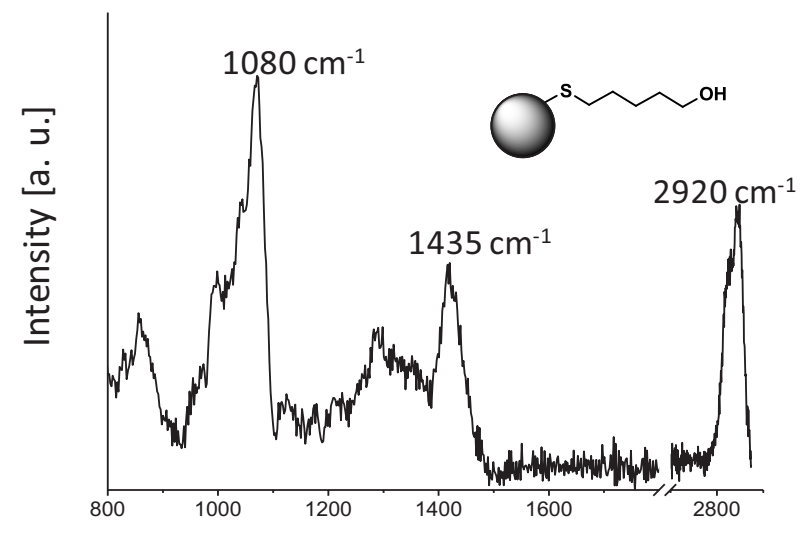

(b)

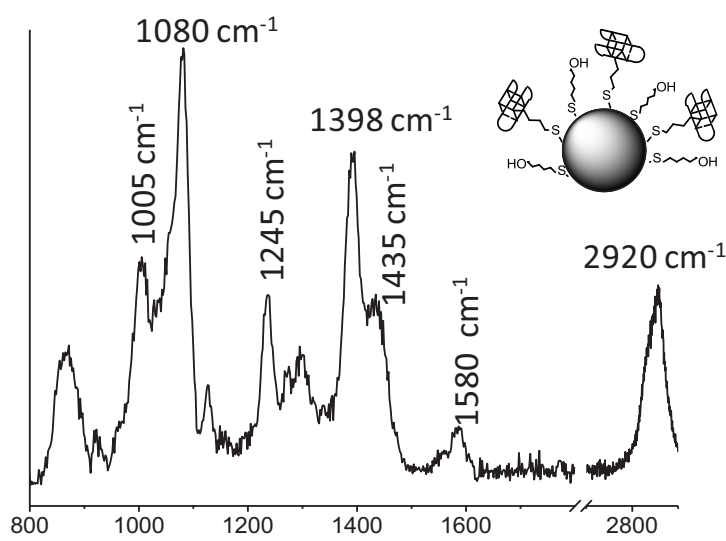

(c)

\section{Shift $\left[\mathrm{cm}^{-1}\right]$}

Fig. 3 SERS spectra of (a) immobilized TBA, (b) blocking agent $(\mathrm{MH})$, and (c) immobilized TBA together with $\mathrm{MH}$. The spectra are normalized with respect to the most intense peak for each trace.

deformation, ${ }^{52}$ which is shifted from the $1425-\mathrm{cm}^{-1}$ band of the spontaneous Raman spectrum, ${ }^{47}$ with some contribution from the ring-stretching mode of the guanine $\left(\sim 1392 \mathrm{~cm}^{-1}\right) .^{50}$ The intensity of the peak suggests there is proximity of this moiety to the metal surface.

Figure 3(c) shows the SERS spectrum of TBA together with MH. It can be seen that the major SERS peaks corresponding to $\mathrm{MH}\left(1080\right.$ and $1435 \mathrm{~cm}^{-1}, \mathrm{C}-\mathrm{C}$ stretch and $\mathrm{C}-\mathrm{H}$ 


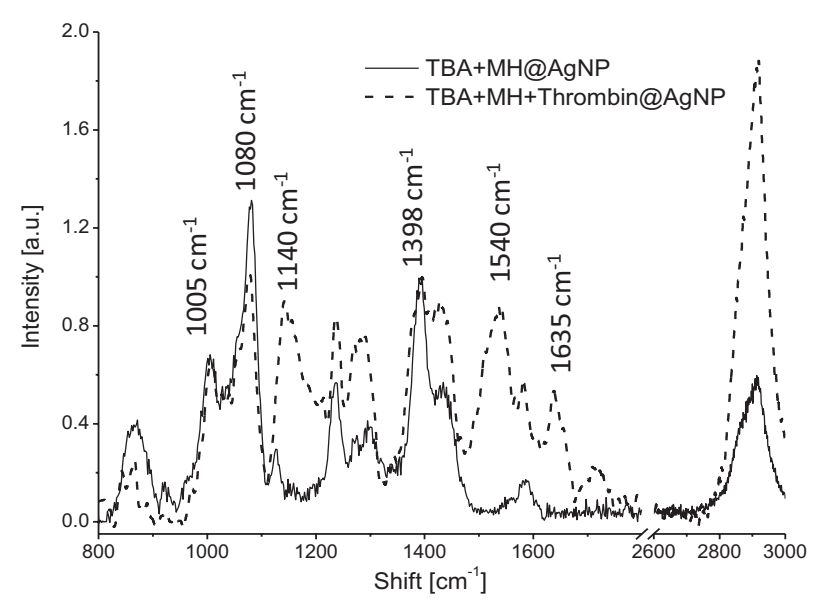

(a)

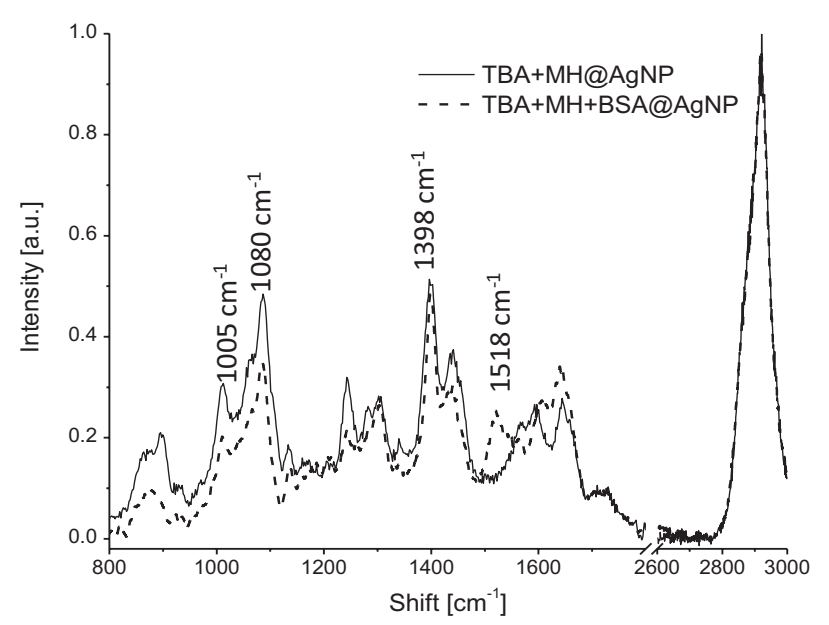

(b)

Fig. 4 (a) SERS of immobilized TBA together with MH (solid line) and TBA with $\mathrm{MH}$ and the target protein, thrombin (dashed line). The spectra normalized with respect to the $1398-\mathrm{cm}^{-1}$ TBA peak. (b) SERS spectra of immobilized TBA with $\mathrm{MH}$ (solid line) and immobilized TBA with $\mathrm{MH}$ and the nonbinding BSA (dashed line). The spectra are normalized with respect to the most intense peak $\left(2920 \mathrm{~cm}^{-1}\right)$ for both.

deformation, respectively) added to the substrate already functionalized with the aptamer are detected together with those peaks corresponding to the aptamer (1005, 1245, and $1398 \mathrm{~cm}^{-1}$ ). The addition of the target protein (thrombin) resulted in additional bands in the spectra [Fig. 4(a)], namely $1635 \mathrm{~cm}^{-1}$ (amide 1, C=O stretch coupled with $\mathrm{C}-\mathrm{N}-\mathrm{H}$ bend), ${ }^{53,54} 1540 \mathrm{~cm}^{-1}$ (amide $2, \mathrm{~N}-\mathrm{H}$ bend coupled with $\mathrm{C}-\mathrm{N}$ stretch), ${ }^{55,56}$ and $1140 \mathrm{~cm}^{-1}(\mathrm{C}-\mathrm{N}$ stretch $),{ }^{54}$ which are protein characteristic vibrational peaks (Table 1). In addition, there is a three-fold increase in the intensity of the $\mathrm{C}-\mathrm{H}$ peak upon addition of thrombin. These observations strongly suggest binding of the target molecule. It may be noted that the amide 2 band is not normally Raman active. However, Raman enhancement near metallic nanoparticles can result in the appearance of vibrational modes that are normally forbidden by selection rules, as well as in the disappearance of certain vibrational bands. This is due to several factors, which include the proximity of the molecular bond to the metal surface and different orientation of the molecule with respect to the metal surface following surface adsorption or binding to capture agent. This may explain the appearance of this band at $1540 \mathrm{~cm}^{-1}$. Similar observations have been reported in the literature. For example, $\mathrm{Hu}$ et al ${ }^{56}$ reported the appearance of an amide 2 band (centered at $1526 \mathrm{~cm}^{-1}$ ) in the SERS spectrum of lysozyme, Kim et al. ${ }^{57}$ observed the presence of this band (at $1520 \mathrm{~cm}^{-1}$ ) in the SERS spectrum of Gly-L-Phe dipeptide, and Podstawka et al. ${ }^{58,59}$ observed this band from 1504 to $1554 \mathrm{~cm}^{-1}$ in the SERS spectra of a series of homoand heterodipeptides.

These spectral changes that were observed with the addition of thrombin to the aptamer functionalized substrate were not detected when the same experiment was conducted using a nonbinding protein (BSA) [Fig. 4(b)]. The spectra of TBA with $\mathrm{MH}$ and of TBA with $\mathrm{MH}$ and BSA are almost superimposable, suggesting that no binding has occurred. However, it can also be seen that there is a noticeable change in the spectrum of TBA with $\mathrm{MH}$, specifically the relative intensities of their major peaks. One possible explanation for this observation is the variation in the amounts of aptamer and mercaptohexanol that were bound to the nanoparticle. The intensity ratio of $1398-\mathrm{cm}^{-1} / 1080-\mathrm{cm}^{-1}$ peak is noticeably higher in this set of spectra, suggesting an increased amount of aptamer adsorbed on the nanoparticle compared to the previous experiment (using thrombin). It is likely that this increase in the amount of aptamer molecules bound to the nanoparticle surface resulted in a higher relative intensity of the $\mathrm{C}-\mathrm{H}$ peak. It is also possible that the higher concentration of bound aptamer led to a change in its orientation with respect to the nanoparticle surface, i.e., from flat or slightly bent to perpendicular, which in turn results in the observed higher relative intensity of the $\mathrm{C}-\mathrm{H}$ peak. ${ }^{60}$ It has been shown both experimentally and theoretically that the $\mathrm{C}-\mathrm{H}$ stretching mode is sensitive to molecule orientation on the nanoparticle surface. ${ }^{61,62}$ In addition to the already mentioned changes in the spectrum of BSA-NP complex, we noted an additional band around $1518 \mathrm{~cm}^{-1}$. One possible explanation for the appearance of this peak is nonspecific binding of BSA or other components (e.g., buffer components) of the test sample to the nanoparticle, resulting from insufficient coverage of the metal surface by both TBA and MH. This observation may indicate that nonspecific binding can be detected and discriminated against based on the different spectral response. While the sensitivity is not yet comparable with commonly used labelbased and sandwiched-type detection methods such as ELISA, it is comparable with other direct detection techniques available in the literature, such as those reported by Jung et al., ${ }^{63}$ a surface acoustic wave (SAW)-based detection of thrombin $(1 \mu \mathrm{M})$ using an aptamer-functionalized sensor chip; and by Neumann et al., ${ }^{15}$ a SERS-based direct detection of target molecules (PDGF and cocaine), wherein the randomness of the SERS spectra of the specific aptamer following exposure to the corresponding target molecule (1 $\mu \mathrm{M}$ PDGF and $20 \mu \mathrm{M}$ cocaine) is monitored. We believe that further improvements (e.g., preconcentration of the sample on the SERS substrate) to the current technique will enhance its sensitivity. 
Pagba et al.: Direct detection of aptamer-thrombin binding via surface-enhanced Raman spectroscopy

Table 1 SERS frequencies of TBA, TBA+MH, and TBA+MH+thrombin and their corresponding vibrational modes. The observed additional peaks on $\mathrm{TBA}+\mathrm{MH}$ and $\mathrm{TBA}+\mathrm{MH}+$ thrombin spectra are listed in bold for clarity.

\begin{tabular}{|c|c|c|c|c|}
\hline \multicolumn{3}{|c|}{ SERS frequency, $\mathrm{cm}^{-1}$} & \multirow[t]{2}{*}{ Assignment } & \multirow[t]{2}{*}{ References } \\
\hline TBA & $\begin{array}{l}\text { TBA } \\
+\mathrm{MH}\end{array}$ & $\begin{array}{l}\mathrm{TBA}+ \\
\mathrm{MH}+ \\
\text { thrombin }\end{array}$ & & \\
\hline 860 & 860 & 860 & sugar vib (C3' endo), & 64 \\
\hline 1005 & 1005 & 1005 & deoxyribose vib & 65 \\
\hline \multirow[t]{3}{*}{$1080(w)$} & $1080(w)$ & $1080(w)$ & $\mathrm{PO}_{2}^{-}$sym str in the TGT loop of TBA & 51 \\
\hline & 1080 & 1080 & $\mathrm{C}-\mathrm{C}$ str in $\mathrm{MH}$ & \\
\hline & & 1140 & $\mathrm{C}-\mathrm{N}$ str of thrombin & 54 \\
\hline 1245 & 1245 & 1245 & $\mathrm{dT}, \mathrm{N}-\mathrm{H}$ def, and $\mathrm{C}-\mathrm{N}$ str of guanine & 66 \\
\hline 1278 & 1278 & 1278 & ring str and $\mathrm{C}-\mathrm{H}$ def of thymine & 67 \\
\hline $1370(w)$ & $1370(w)$ & $1370(w)$ & dT, dG C2' endo/syn & 66 \\
\hline \multirow[t]{3}{*}{1398} & 1398 & 1398 & deoxyribosyl $\left(\mathrm{C}^{\prime} \mathrm{H}_{2}\right)$ def & $52,65,66$, and 68 \\
\hline & 1435 & 1435 & $\mathrm{C}-\mathrm{H}$ def of $\mathrm{MH}$ & \\
\hline & & 1540 & Amide 2 of protein & 55 and 56 \\
\hline \multirow[t]{2}{*}{1580} & 1580 & 1580 & $\mathrm{C} 2=\mathrm{N} 3$ of guanine & \\
\hline & & 1635 & Amide I of protein & 53 and 54 \\
\hline $1660(w)$ & $1660(w)$ & $1660(w)$ & $\mathrm{C} 6=\mathrm{O} 6(\mathrm{DNA})$ & \\
\hline 2920 & 2920 & 2920 & deoxyribosyl C-H str & 47 \\
\hline
\end{tabular}

\section{Conclusions}

We demonstrate a simple and selective SERS-based method for direct detection of biomolecules based on molecular recognition, which require fewer steps than traditional sandwiched-based assays. Binding of the target molecule to the molecular receptor (aptamer) is manifested by the appearance of the molecule's characteristic vibrational bands $(\mathrm{C}-\mathrm{N}$ stretching, amide 1 and amide 2 bands), in addition to the vibrational peaks corresponding to the receptor and blocking agent. It is important to note that interfering species that are able to bind may produce a noticeably different spectral response, thus further improving the selectivity.

Since aptamers are available not only for biomolecules but also for small molecules such as ricin, trinitrotoluene, and polycyclic aromatic hydrocarbons, this technique can be useful for the detection not only of medical biomarkers but also of environmental pollutants, as well as biological and chemical threat agents. In addition, the SERS substrates can be functionalized with different aptamers, allowing for multiplexed screening and detection. The simplicity of the developed label-free detection technique could be further utilized for on-chip and real-time detection by implementing it on a nano- and microscale platform.

\section{Acknowledgments}

This work is supported by funding from the National Science Foundation (NSF) and the Laboratory Directed Research and Development Program of Lawrence Livermore National Laboratory (LLNL). Cho acknowledges (LLNL-JRNL) for a Lawrence Scholar Program predoctoral fellowship (LLNLJRNL-421298-DRAFT). The Center for Biophotonics Science and Technology is a designated NSF Science and Technology Center, managed by the University of California, Davis, under Cooperative Agreement number PHY 0120999. We would like to thank Rick Moerschell formerly of Biorad Incorporated for useful discussions.

\section{References}

1. Y. C. Cao, R. Jin, J. M. Nam, C. S. Thaxton, and C. A. Mirkin, "Raman dye-labeled nanoparticle probes for proteins," J. Am. Chem. Soc. 125(48), 14676-14677 (2003).

2. J. M. Nam, C. S. Thaxton, and C. A. Mirkin, "Nanoparticle-based bio-bar codes for the ultrasensitive detection of proteins," Science 301(5641), 1884-1886 (2003).

3. X. X. Han, L. J. Cai, J. Guo, C. X. Wang, W. D. Ruan, W. Y. Han, W. Q. Xu, B. Zhao, and Y. Ozaki, "Fluorescein isothiocyanate linked immunoabsorbent assay based on surface-enhanced resonance Raman scattering," Anal. Chem. 80(8), 3020-3024 (2008). 
4. W. Zhao, M. A. Brook, and Y. Li, "Design of gold nanoparticle-based colorimetric biosensing assays," ChemBioChem 9(15), 2363-2371 (2008).

5. V. Pavlov, Y. Xiao, B. Shlyahovsky, and I. Willner, "Aptamerfunctionalized $\mathrm{Au}$ nanoparticles for the amplified optical detection of thrombin," J. Am. Chem. Soc. 126(38), 11768-11769 (2004).

6. M. Sha, S. Penn, G. Freeman, and W. Doering, "Detection of human viral RNA via a combined fluorescence and SERS molecular beacon assay," NanoBiotechnology 3(1), 23-30 (2007).

7. B. Dubertret, M. Calame, and A. J. Libchaber, "Single-mismatch detection using gold-quenched fluorescent oligonucleotides," Nat. Biotechnol. 19(4), 365-370 (2001).

8. S. R. Kalb and J. R. Barr, "Mass spectrometric detection of ricin and its activity in food and clinical samples," Anal. Chem. 81(6), 20372042 (2009).

9. W. E. Doering and S. Nie, "Single-molecule and single-nanoparticle SERS: examining the roles of surface active sites and chemical enhancement," J. Phys. Chem. B 106(2), 311-317 (2002).

10. M. Moskovits, "Surface-enhanced Raman spectroscopy: a brief retrospective," J. Raman Spectrosc. 36(6-7), 485-496 (2005).

11. A. Otto, "The 'chemical' (electronic) contribution to surfaceenhanced Raman scattering," J. Raman Spectrosc. 36(6-7), 497-509 (2005).

12. K. Hering, D. Cialla, K. Ackermann, T. Dörfer, R. Möller, H. Schneidewind, R. Mattheis, W. Fritzsche, P. Rösch, and J. Popp, "SERS: a versatile tool in chemical and biochemical diagnostics," Anal. Bioanal. Chem. 390(1), 113-124 (2008).

13. M. D. Porter, R. J. Lipert, L. M. Siperko, G. Wang, and R. Narayanana, "SERS as a bioassay platform: fundamentals, design, and applications," Chem. Soc. Rev. 37(5), 1001-1011 (2008).

14. H. Cho, B. R. Baker, S. Wachsmann-Hogiu, C. V. Pagba, T. A. Laurence, S. M. Lane, L. P. Lee, and J. B. H. Tok, "Aptamer-based SERRS sensor for thrombin detection," Nano Lett. 8(12), 4386-4390 (2008).

15. O. Neumann, D. Zhang, F. Tam, S. Lal, P. Wittung-Stafshede, and N. J. Halas, "Direct optical detection of aptamer conformational changes induced by target molecules," Anal. Chem. 81(24), 10002-10006 (2009).

16. A. D. Ellington and J. W. Szostak, "In vitro selection of RNA molecules that bind specific ligands," Nature 346(6287), 818-822 (1990).

17. N. de-los-Santos-Álvarez, M. A. J. Lobo-Castañón, A. J. MirandaOrdieres, and P. Tuñón-Blanco, "Aptamers as recognition elements for label-free analytical devices," TrAC, Trends Anal. Chem. 27(5), 437-446 (2008).

18. T. Hermann and D. J. Patel, "Adaptive recognition by nucleic acid aptamers," Science 287(5454), 820-825 (2000).

19. J. X. N. S. Y. Y. Jijun Tang, "The DNA aptamers that specifically recognize ricin toxin are selected by two in vitro selection methods," Electrophoresis 27(7), 1303-1311 (2006).

20. J. G. Bruno and J. L. Kiel, "In vitro selection of DNA aptamers to anthrax spores with electrochemiluminescence detection," Biosens. Bioelectron. 14(5), 457-464 (1999).

21. E. Ehrentreich-Förster, D. Orgel, A. Krause-Griep, B. Cech, V. Erdmann, F. Bier, F. Scheller, and M. Rimmele, "Biosensor-based on-site explosives detection using aptamers as recognition elements," Anal. Bioanal. Chem. 391(5), 1793-1800 (2008).

22. B. J. Hicke, C. Marion, Y. F. Chang, T. Gould, C. K. Lynott, D. Parma, P. G. Schmidt, and S. Warren, "Tenascin-C aptamers are generated using tumor cells and purified protein," J. Biol. Chem. 276(52), 48644-48654 (2001).

23. C. Wang, M. Zhang, G. Yang, D. Zhang, H. Ding, H. Wang, M. Fan, B. Shen, and N. Shao, "Single-stranded DNA aptamers that bind differentiated but not parental cells: subtractive systematic evolution of ligands by exponential enrichment," J. Biotechnol. 102(1), 15-22 (2003).

24. J. Ruckman, L. S. Green, J. Beeson, S. Waugh, W. L. Gillette, D. D. Henninger, L. Claesson-Welsh, and N. Janjic, " 2 '-fluoropyrimidine RNA-based aptamers to the 165-amino acid form of vascular endothelial growth factor (VEGF165). Inhibition of receptor binding and vegf-induced vascular permeability through interactions requiring the exon 7-encoded domain," J. Biol. Chem. 273(32), 20556-20567 (1998).

25. C. H. B. Chen, G. A. Chernis, V. Q. Hoang, and R. Landgraf, "Inhibition of heregulin signaling by an aptamer that preferentially binds to the oligomeric form of human epidermal growth factor receptor3," Proc. Natl. Acad. Sci. U.S.A. 100(16), 9226-9231 (2003).

26. N. Sayer, J. Ibrahim, K. Turner, A. Tahiri-Alaoui, and W. James, "Structural characterization of a 2'F-RNA aptamer that binds a HIV-1 SU glycoprotein, gp120," Biochem. Biophys. Res. Commun. 293(3), 924-931 (2002).

27. R. Yamamoto and P. K. R. Kumar, "Molecular beacon aptamer fluoresces in the presence of Tat protein of HIV-1," Genes Cells 5(5), 389-396 (2000).

28. O. Kensch, B. A. Connolly, H. J. r. Steinhoff, A. McGregor, R. S. Goody, and T. Restle, "HIV-1 reverse transcriptase-pseudoknot RNA aptamer interaction has a binding affinity in the low picomolar range coupled with high specificity," J. Biol. Chem. 275(24), 18271-18278 (2000).

29. N. Jing, R. F. Rando, Y. Pommier, and M. E. Hogan, "Ion selective folding of loop domains in a potent anti-HIV oligonucleotide," Biochemistry 36(41), 12498-12505 (1997).

30. S. Tombelli, M. Minunni, and M. Mascini, "Aptamers-based assays for diagnostics, environmental and food analysis," Biomol. Eng. 24(2), 191-200 (2007).

31. W. Yoshida, E. Mochizuki, M. Takase, H. Hasegawa, Y. Morita, H. Yamazaki, K. Sode, and K. Ikebukuro, "Selection of DNA aptamers against insulin and construction of an aptameric enzyme subunit for insulin sensing," Biosens. Bioelectron. 24(5), 1116-1120 (2009).

32. A. C. Connor, K. A. Frederick, E. J. Morgan, and L. B. McGown, "Insulin capture by an insulin-linked polymorphic region G-quadruplex DNA oligonucleotide," J. Am. Chem. Soc. 128(15), 4986-4991 (2006).

33. T. Wiegand, P. Williams, S. Dreskin, M. Jouvin, J. Kinet, and D. Tasset, "High-affinity oligonucleotide ligands to human $\operatorname{IgE}$ inhibit binding to Fc epsilon receptor I," J. Immunol. 157(1), 221-230 (1996).

34. L. C. Bock, L. C. Griffin, J. A. Latham, E. H. Vermaas, and J. J. Toole, "Selection of single-stranded DNA molecules that bind and inhibit human thrombin," Nature 355(6360), 564-566 (1992).

35. S. Song, L. Wang, J. Li, C. Fan, and J. Zhao, "Aptamer-based biosensors," TrAC, Trends Anal. Chem. 27(2), 108-117 (2008).

36. T. Mairal, V. Cengiz Özalp, P. Lozano Sánchez, M. Mir, I. Katakis, and C. O'Sullivan, "Aptamers: molecular tools for analytical applications," Anal. Bioanal. Chem. 390(4), 989-1007 (2008).

37. S. Tombelli, M. Minunni, and M. Mascini, "Analytical applications of aptamers," Biosens. Bioelectron. 20(12), 2424-2434 (2005).

38. M. Famulok, J. S. Hartig, and G. Mayer, "Functional aptamers and aptazymes in biotechnology, diagnostics, and therapy," Chem. Rev. 107(9), 3715-3743 (2007).

39. L. R. Paborsky, S. N. McCurdy, L. C. Griffin, J. J. Toole, and L. L. Leung, "The single-stranded DNA aptamer-binding site of human thrombin," J. Biol. Chem. 268(28), 20808-20811 (1993).

40. K. Y. Wang, S. McCurdy, R. G. Shea, S. Swaminathan, and P. H. Bolton, "A DNA aptamer which binds to and inhibits thrombin exhibits a new structural motif for DNA," Biochemistry 32(8), 1899_ 1904 (1993).

41. K. Y. Wang, S. H. Krawczyk, N. Bischofberger, S. Swaminathan, and P. H. Bolton, "The tertiary structure of a DNA aptamer which binds to and inhibits thrombin determines activity," Biochemistry 32(42), 11285-11292 (1993).

42. R. F. Macaya, P. Schultze, F. W. Smith, J. A. Roe, and J. Feigon, "Thrombin-binding DNA aptamer forms a unimolecular quadruplex structure in solution," Proc. Natl. Acad. Sci. U.S.A. 90(8), 3745-3749 (1993).

43. P. Schultze, R. F. Macaya, and J. Feigon, "Three-dimensional solution structure of the thrombin-binding DNA aptamer d(GGTTGGTGTGGTTGG)," J. Mol. Biol. 235(5), 1532-1547 (1994).

44. K. Padmanabhan, K. P. Padmanabhan, J. D. Ferrara, J. E. Sadler, and A. Tulinsky, "The structure of alpha-thrombin inhibited by a $15-\mathrm{mer}$ single-stranded DNA aptamer," J. Biol. Chem. 268(24), 17651-17654 (1993).

45. P. Alberti and J. L. Mergny, "DNA duplex-quadruplex exchange as the basis for a nanomolecular machine," Proc. Natl. Acad. Sci. U.S.A. 100(4), 1569-1573 (2003).

46. P. C. Lee and D. Meisel, "Adsorption and surface-enhanced Raman of dyes on silver and gold sols," J. Phys. Chem. 86(17), 3391-3395 (1982).

47. C. V. Pagba, S. M. Lane, and S. Wachsmann-Hogiu, "Raman and surface-enhanced Raman spectroscopic studies of the 15-mer DNA 
thrombin-binding aptamer," J. Raman Spectrosc. 41(3), 241-247 (2010).

48. T. Miura and G. J. Thomas, "Structural polymorphism of telomere DNA: interquadruplex and duplex-quadruplex conversions probed by Raman spectroscopy," Biochemistry 33(25), 7848-7856 (1994).

49. J. M. Benevides, A. H. J. Wang, G. A. Van der Marel, J. H. Van Boom, and G. J. Thomas, "Crystal and solution structures of the B-DNA dodecamer d(CGCAAATTTGCG) probed by Raman spectroscopy: heterogeneity in the crystal structure does not persist in the solution structure," Biochemistry 27(3), 931-938 (1988).

50. Y. Nishimura, M. Tsuboi, T. Sato, and K. Aoki, "Conformationsensitive Raman lines of mononucleotides and their use in a structure analysis of polynucleotides: guanine and cytosine nucleotides," $J$. Mol. Struct. 146, 123-153 (1986).

51. J. A. Mondragon-Sanchez, J. Liquier, R. H. Shafer, and E. Taillandier, "Tetraplex structure formation in the thrombin-binding DNA aptamer by metal cations measured by vibrational spectroscopy," $J$. Biomol. Struct. Dyn. 22(3), 365-373 (2004).

52. K. Kneipp and J. Flemming, "Surface enhanced Raman scattering (SERS) of nucleic acids adsrobed on colloidal silver particles," $J$. Mol. Struct. 145(1-2), 173-179 (1986).

53. W. L. Peticolas, "Raman spectroscopy of DNA and proteins," Methods Enzymol. 246, 389-416 (1995).

54. M. C. Chen, R. C. Lord, and R. Mendelsohn, "Laser-excited Raman spectroscopy of biomolecules. V. Conformational changes associated with the chemical denaturation of lysozyme," J. Am. Chem. Soc. 96(10), 3038-3042 (1974).

55. Y. P. Zhang, R. N. A. H. Lewis, R. S. Hodges, and R. N. McElhaney, "FTIR spectroscopic studies of the conformation and amide hydrogen exchange of a peptide model of the hydrophobic transmembrane .alpha.-helixes of membrane proteins," Biochemistry 31(46), 11572 11578 (1992).

56. J. Hu, R. S. Sheng, Z. S. Xu, and Y. Zeng, "Surface enhanced Raman spectroscopy of lysozyme," Spectrochim. Acta, Part A 51(6), 10871096 (1995).

57. S. K. Kim, M. S. Kim, and S. W. Suh, "Surface-enhanced Raman scattering (SERS) of aromatic amino acids and their glycyl dipeptides in silver sol," J. Raman Spectrosc. 18(3), 171-175 (1987).
58. E. Podstawka, Y. Ozaki, and L. M. Proniewicz, "Part I: surfaceenhanced Raman spectroscopy investigation of amino acids and their homodipeptides adsorbed on colloidal silver," Appl. Spectrosc. 58(5), 570-580 (2004).

59. E. Podstawka, Y. Ozaki, and L. M. Proniewicz, "Part II: surfaceenhanced Raman spectroscopy investigation of methionine containing heterodipeptides adsorbed on colloidal silver," Appl. Spectrosc. 58(5), 581-590 (2004).

60. A. Barhoumi, D. Zhang, F. Tam, and N. J. Halas, "Surface-enhanced Raman spectroscopy of DNA," J. Am. Chem. Soc. 130(16), 55235529 (2008).

61. M. Moskovits and J. S. Suh, "Surface selection rules for surfaceenhanced Raman spectroscopy: calculations and application to the surface-enhanced Raman spectrum of phthalazine on silver," J. Phys. Chem. 88(23), 5526-5530 (1984).

62. X. Gao, J. P. Davies, and M. J. Weaver, "Test of surface selection rules for surface-enhanced Raman scattering: the orientation of adsorbed benzene and monosubstituted benzenes on gold," J. Phys. Chem. 94(17), 6858-6864 (1990).

63. A. Jung, T. M. A. Gronewold, M. Tewes, E. Quandt, and P. Berlin, "Biofunctional structural design of SAW sensor chip surfaces in a microfluidic sensor system," Sens. Actuators B 124(1), 46-52 (2007).

64. W. L. Peticolas and E. Evertsz, "Conformation of DNA in vitro and in vivo from laser Raman scattering," Methods Enzymol. 211, 335-352 (1992).

65. W. S. G. J. T. B. Prescott, Jr., "Characterization of DNA structures by laser Raman spectroscopy,” Biopolymers 23(2), 235-256 (1984).

66. C. Krafft, J. M. Benevides, and G. J. Thomas, Jr., "Secondary structure polymorphism in Oxytricha nova telomeric DNA," Nucleic Acids Res. 30(18), 3981-3991 (2002).

67. C. Otto, T. J. J. van den Tweel, F. F. M. de Mul, and J. Greve, "Surface-enhanced Raman spectroscopy of DNA bases," J. Raman Spectrosc. 17(3), 289-298 (1986).

68. J. M. Benevides and G. J. Thomas, Jr., "Characterization of DNA structures by Raman spectroscopy: high-salt and low-salt forms of double helical poly (dG-dC) in $\mathrm{H} 2 \mathrm{O}$ and $\mathrm{D} 2 \mathrm{O}$ solutions and application to B, Z and A-DNA*," Nucleic Acids Res. 11(16), 5747-5761 (1983). 